\title{
Thinking outside the lungs: 'other' risk factors for pneumonia hospitalisation
}

\section{I Bloom, J K Quint}

The symptoms of pneumonic infection can be traced back through history; first being described during early Greek civilisation. But it wasn't until the 19th century the disorder was described in its own right, once causative bacteria were observed. Although mortality rates then rapidly declined, with the mass production of antibiotics, today it still remains a serious global problem. ${ }^{1}$

At the bedside, several determinants are now well recognised as severity markers for community-acquired pneumonia (CAP), such as age $>65$ years, COPD, smoking and dementia. ${ }^{2}$ In addition, we all look out for immunosuppressive treatments, and appreciate infection is often more severe in people who are immunocompromised, but other factors, such as androgen-deprivation therapy (ADT) may not usually be considered.

Respiratory clinicians are highly likely to treat patients with prostate cancer, the second most common cancer in the UK with a rapidly growing incidence (147/ 10000 in 2014, predicted to be 233/ 100000 in 2035). ${ }^{3}$ As prostate cancer is androgen-dependent a common form of treatment in those with metastatic or high-risk disease has been the use of ADT. $^{4}$ Recently, it is increasingly being used in patients with less advanced disease, although without clear evidence of benefit. ${ }^{5}$ The list of side effects from prolonged exposure is long and worrying, including osteoporosis, metabolic syndrome, sexual dysfunction and cardiovascular harm, and has thus led to the frequent use of intermittent ADT therapy, but with questionable success. ${ }^{6}$

Two prior observational studies have addressed the issue of pneumonia in association with ADT; these limited studies found conflicting results, with one limited to only one type of ADT and the other suffering from immortal time bias and an underestimation of the association. ${ }^{7} 8$

Department of Respiratory Epidemiology, Occupational Medicine and Public Health, National Heart and Lung Institute, Imperial College London, London, UK

Correspondence to Dr J K Quint, Department of Respiratory Epidemiology, Occupational Medicine and Public Health, National Heart and Lung Institute, Emmanuel Kaye Building, Imperial College London, London SW3 6LR, UK; j.quint@imperial.ac.uk
In Thorax, Hicks et $a l^{9}$ carried out a large-scale, population-based study of UK men with newly diagnosed non-metastatic prostate cancer between 1998 and 2015. They used data obtained from electronic healthcare records to comprehensively measure the risk of hospitalisation for CAP, comparing patients currently being treated with ADT, or patients who had past ADT use, to patients who had never received ADT. All patients on any current form of ADT had an increased adjusted risk of $81 \% \quad(\mathrm{HR}=1.81,95 \%$ CI 1.47 to 2.23); patients taking stand-alone gonadotropin-releasing hormone agonists (the majority of prescribed ADT) had a $73 \%$ increased risk $(\mathrm{HR}=1.73,95 \% \mathrm{CI}$ 1.39 to 2.16 ). The association was evident with less than 6 months use of ADT, which rose to a maximum risk with 13-18 months treatment, but patients with past ADT use did not have an increased risk. The possible mechanisms explaining ADT's potentially immunosuppressive effects include interference with $\mathrm{T}$ cell priming ${ }^{10}$ and impairment of granulopoiesis. $^{11}$

This was a well conducted study with convincing findings that the authors backed up with credible immunological reasons. This study is also a timely reminder of both the power and potential weaknesses of real world evidence. The data source used in this study (Clinical Practice Research Datalink) has several strengths, including its huge size (coverage of over 11.3 million patients), breadth of coverage, long-time follow-up, representativeness and data quality, which are all successfully exploited by this study. ${ }^{12}$ However, there are other aspects of such data sources, if not carefully preconsidered, that can lead to flawed conclusions. One such limitation is that some aspects of health may not be recorded, such as prescriptions in secondary care; this could potentially lead to exposure misclassification, although the authors argue here that they believe that to be limited. They point out that there is a high prevalence of ADT use in this cohort (59\%), which maybe a reasonable argument, but one that is hard to prove either way from available information.

Another unrecorded variable was cancer stage and grade. The authors adjusted for multiple potential confounders but were unable to adjust for disease severity. They employed two alternative statistical models (high dimensional propensity scoring and marginal structure models) that can be used in observational studies to overcome unmeasured confounding effects. The development of clinical data analysis expertise is rapidly progressing, chasing the profligate amassing of patient data, and ever increases the value of these real world data studies.

There is currently little evidence base for commencing ADT in patients with low-risk prostate cancer, yet it happens commonly. This study, on the other hand, provides sufficient everyday evidence to ponder that ADT might well be an overlooked determinant of hospitalisation for CAP, and that this apparent association, with an increasingly prevalent treatment, warrants further epidemiological investigation.

Contributors CIB wrote the first draft. JKQ and CIB contributed to subsequent drafts and approved the version submitted.

\section{Competing interests None declared.}

Provenance and peer review Commissioned; externally peer reviewed.

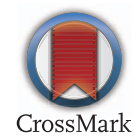

To cite Bloom Cl, Quint JK. Thorax 2017;72:596-597.

Published Online First 7 March 2017



http://dx.doi.org/10.1136/thoraxjnl-2016-209512

Thorax 2017;72:596-597.

doi:10.1136/thoraxjnl-2016-209773

\section{REFERENCES}

1 World Health Organization. World Pneumonia Day 2014: Pneumonia Fact Sheet. Geneva: WHO, 2014 http://worldpneumoniaday.org/wp-content/uploads/ 2014/11/Final-WPD-2014-Fact-Sheet1.pdf.

2 Torres A, Peetermans WE, Viegi G, et al. Risk factors for community-acquired pneumonia in adults in Europe: a literature review. Thorax 2013;68:1057-65

3 Cancer Research UK. Prostate cancer statistics. London: Cancer Research UK, 2014. http://www. cancerresearchuk.org/health-professional/cancerstatistics/statistics-by-cancer-type/prostatecancer\#heading-Zero

4 National Institute for Health and Care Excellence (NICE). Prostate cancer: diagnosis and management [CG175]. London: NICE, Jan 2014.

5 Gilbert SM, Kuo YF, Shahinian VB. Prevalent and incident use of androgen deprivation therapy among men with prostate cancer in the United States. Urol Oncol 2011:29:647-53.

6 Liede A, Hallett DC, Hope K, et al. International survey of androgen deprivation therapy (ADT) for 
non-metastatic prostate cancer in 19 countries. ESMO Open 2016;1:e000040.

7 Schmid M, Hanske J, Ravi P, et al. Relationship between androgen deprivation therapy and community-acquired respiratory infections in patients with prostate cancer. Int J Urol 2016;23:305-11.

8 Chung SD, Liu SP, Lin HC, et al. Increased risk of pneumonia in patients receiving gonadotropin-releasing hormone agonists for prostate cancer. PLOS ONE 2014:9:e101254.

9 Hicks BM, Yin H, Bladou F, et al. Androgen deprivation therapy for prostate cancer and the risk of hospitalisation for community-acquired pneumonia. Thorax 2017;72:628-34.

10 Pu Y, Xu M, Liang Y, et al. Androgen receptor antagonists compromise $T$ cell response against prostate cancer leading to early tumor relapse.
Sci Transl Med 2016;8:333ra47.

11 Chuang KH, Altuwaijri S, Li G, et al. Neutropenia with impaired host defense against microbial infection in mice lacking androgen receptor. J Exp Med 2009;206:1181-99.

12 Herrett E, Gallagher AM, Bhaskaran K, et al. Data resource profile: clinical practice research datalink (CPRD). Int J Epidemiol 2015;44:827-36. 